\title{
Bidirectional contrast, matching, and power functions obtained in sucrose consumption by rats
}

\author{
CHARLES F. FLAHERTY and STEVEN J. SEPANAK \\ Rutgers University, New Brunswick, New Jersey 08902
}

\begin{abstract}
Rats were given alternating 1-min access to two tubes containing sucrose solutions that varied in concentraton $(32 \%$ vs. $2 \%, 32 \%$ vs. $4 \%, 32 \%$ vs. $8 \%$, and $32 \%$ vs. $16 \%)$. Lick rate for $32 \%$ sucrose was higher when the alternative tube contained a lower concentration solution than when both tubes contained $32 \%$ (a positive-contrast effect), and lick rate for the lower concentration solution $(2 \%, 4 \%, 8 \%$, or $16 \%)$ was lower when the alternative tube contained $32 \%$ than when both tubes contained the lower concentration solution (negative contrast effect). Proportion of licks made for $32 \%$ under contrast conditions tended to match the proportion of concentration available from that tube. Regression analysis of the ratio of licks made to the two tubes under contrast conditions as a function of ratio of concentrations available indicated a good fit to a power function with an exponent of 1.13 , within the range of those typically found in human magnitude estimation studies of relative sweetness.
\end{abstract}

The influence of contextual stimuli on the perception of focal stimuli is a well-established fact of behavior. For example, the apparent brightness, hue, or weight of a given object is influenced by concurrent or immediate prior experience with objects of a different brightness, hue, or weight (Coren \& Brussel, 1973; DiLollo, 1964; Helson, 1964; Ratliff, 1971). These effects of context on perception are termed contrast effects, where contrast means an exaggeration of stimulus difference as a function of context.

Apparently analogous contrast effects are also found when different values of reward are used in the investigation of animal behavior. For example, animals shifted from a large to a small reward usually perform more poorly for the small reward than do animals that have experienced only the lower level of reward (Crespi, 1942; Mellgren, 1972), a result termed a negative contrast effect. Conversely, animals shifted from a small to a large reward will sometimes perform at a higher level for the large reward than will animals that have experienced only the large reward (Crespi, 1942; Mellgren, 1972), an outcome termed a positive contrast effect.

The present paper is concerned with contrast obtained in the consumption of sucrose solutions. In previous papers, we have described a paradigm in which reliable bidirectional contrast effects may be obtained (Flaherty \& Avdzej, 1974; Flaherty \&

This research was supported by a grant from the Rutgers Research Council and by a Biological Research Support grant. Portions of these data were presented at the 1974 Psychonomic Society meetings.
Largen, 1975; Flaherty \& Lombardi, 1977). The procedure we used in these experiments was one of allowing rats $1-$ min periods of access to two drinking tubes which contained sucrose solutions of different concentration (32\% and $4 \%$ ). On some test days both tubes contained the same solution (either $4 \%$ or $32 \%$ ), on other days one tube contained $32 \%$ and the other contained the $4 \%$ solution. Using this procedure, we have found that rats reliably lick more for the $32 \%$ solution under comparison conditions (32 vs. 4) than under noncomparison conditions (both tubes containing $32 \%$ ), and reliably less for the $4 \%$ solution under comparison conditions than under noncomparison conditions. These effects have been found with both between-subject and within-subject control conditions and with measures of intake and latency to initiate drinking, as well as with a lick-rate measure (Flaherty \& Largen, 1975).

The present paper is concerned with data obtained when the concentrations of the two sucrose solutions presented to the rats were systematically varied.

\section{METHOD}

Subjects

Twelve naive, male Sprague-Dawley rats, approximately 150 days old and purchased from Carworth Labs, were used as subjects. The rats were deprived to $80 \%$ of their free-feeding weights and maintained at that level by once-per-day feeding. Water was continuously available in the home cage. The rats were maintained on a 14-h-on, 10-h-off light cycle, and tested approximately half-way through the light-on portion of this cycle.

\footnotetext{
Apparatus

Testing was conducted in three identical Plexiglas chambers measuring $30 \times 25 \times 25 \mathrm{~cm}$. On one side of the chamber there
} 
were two centrally located 1.5 -cm-diam holes spaced $21.7 \mathrm{~cm}$ apart and $4 \mathrm{~cm}$ above the wire-mesh floor. Two graduated cylinders, located outside the chamber, were programmed so that either cylinder could automatically be moved into a drinking position in which the orifice of the drinking spout was centered in the 1.5-cm-diam hole, flush with the outside wall of the chamber. Pilot lights, mounted on either side of the chamber, close to the drinking access holes, were illuminated whenever the cylinder in closest proximity to that light was in the drinking position. A Contact-relay circuit was used to measure the licking response.

\section{Procedure}

On each test day, the rats were placed in the apparatus containing two spatially separated drinking tubes. The tubes were available one at a time, always starting with the left tube, and alternated after the rat had a 1-min access period, beginning with the first lick. Each test session continued until the rat had three 1-min periods of access to each tube. Contrast was investigated by varying the concentration of sucrose solutions available in the two tubes across days. With a given pair of concentrations, e.g., $32 \%$ vs. $2 \%$, the animals were presented with a 4-day sequence. On one day both tubes contained $32 \%$ and on another day both contained $2 \%$; on one of the two remaining days the left tube contained $2 \%$ and right tube $32 \%$, whereas on the other day the positions were reversed. The actual order of these conditions was randomized in each 4-day cycle. Positive contrast was measured by examining lick rates for the $32 \%$ solution when the alternative tube contained the $2 \%$ solution as compared to when both tubes contained $32 \%$. Similarly, negative contrast was examined by measuring lick rates for the $2 \%$ solution when the alternative tube contained $32 \%$ sucrose as compared to when both tubes contained $2 \%$ sucrose. These comparisons were made with measures of lick rate, and latency to initiate drinking obtained for each minute of the daily sessions.

In the analyses presented below, the data from the last $4 \mathrm{~min}$ only of each condition were included. These values were obtained as follows. On control days (when both tubes contained the same solution), the mean lick rate was obtained simply over the last 4 min of a daily session. On contrast days (when the tubes contained different solutions), these values were obtained by averaging across days in order to counterbalance for the location of the differential concentrations. For example, in a $32-4$ cycle, the mean for $32 \%$ under contrast conditions was obtained by averaging the lick rates for the $3 \mathrm{rd}$ and 5 th min when $32 \%$ was on the left, with the lick rates obtained in the 4th and 6th min when the $32 \%$ was on the right.

Each rat was exposed to this 4-day cycle of contrast and control conditions a total of four times, each time with a different concentration of sucrose as the lower value, but with $32 \%$ always as the higher concentration. The values used for the lower concentration were $2 \%, 4 \%, 8 \%$, and $16 \%$. The order in which these different pairs were presented was varied according to a 4 by 4 Latin square plan, and the square was repeated three times with four subjects per square. There was a 3-day break, during which the rats were maintained at their deprivation weights, between each 4-day test cycle.

Sucrose solutions were prepared by weight [solute/(solute + solvent)] from commercial grade cane sugar and tap water. The solutions were mixed at least $24 \mathrm{~h}$ in advance of a test session and were presented at room temperature.

\section{RESULTS}

The data presented in Figure 1 represent the mean lick rates obtained over the last $4 \mathrm{~min}$ of each 6-min exposure to a pair of concentrations. The two

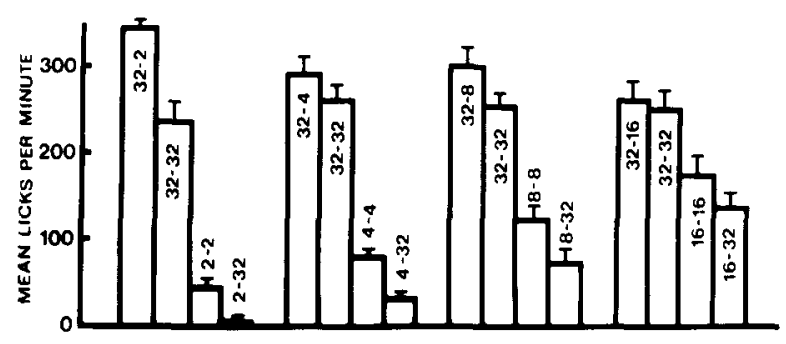

Figure 1. Mean lick rates and standard errors obtained with different sucrose-concentration pairing conditions; 32-2 indicates $32 \%$ available from one tube and $2 \%$ available from the alternative tube, etc.

middle bars in each set of four represent data obtained from control conditions in which both tubes available within a session contained the same solution. The extreme bars within each set of four represent lick rates obtained under contrast conditions, i.e., when the two tubes contained disparate sucrose solutions.

It is apparent from Figure 1 that, with each pair of concentrations, lick rates obtained under comparison conditions (different solutions in the two tubes) showed more exaggerated concentration effects than lick rates under noncomparison conditions. The rats licked reliably more of the $32 \%$ solution when it was paired with each of the lower concentrations than they did when both tubes contained $32 \%[\mathrm{~F}(1,77)=15.84, \mathrm{p}<.001]$, and reliably less for each of the lower concentrations when paired with the $32 \%$ solution than they did when both tubes contained the lower concentration solution $[\mathrm{F}(1,77)=20.79, \mathrm{p}<.001]$. In addition, value of the lower concentration itself had an effect; the higher the concentration, the greater the lick rate $[F(1,77)=37.70, p<.001]$.

It has proven interesting to examine the present data in terms other than the standard contrast analysis presented above. In Figure 2, the data from the contrast conditions (days on which each tube contained different concentrations) are presented in terms of proportions. Plotted on the abscissa is the proportion of total "concentration" available in the $32 \%$ tube across the four pairings. For example, on days when one tube contained $32 \%$ and the alternative contained $16 \%$ sucrose, the $32 \%$ tube would contain .67 $[32 /(32+16)]$ of the total "concentration" available. Plotted on the ordinate is the proportion of licks the animals made from the tube containing the higher concentration as a function of the different pairs of concentrations presented. It is apparent that proportion of licks very nearly matches the proportion of concentration available.

It has been suggested (Baum, 1974) that similar matching functions obtained in operant tasks (e.g., Herrnstein, 1970) can be considered as special cases 


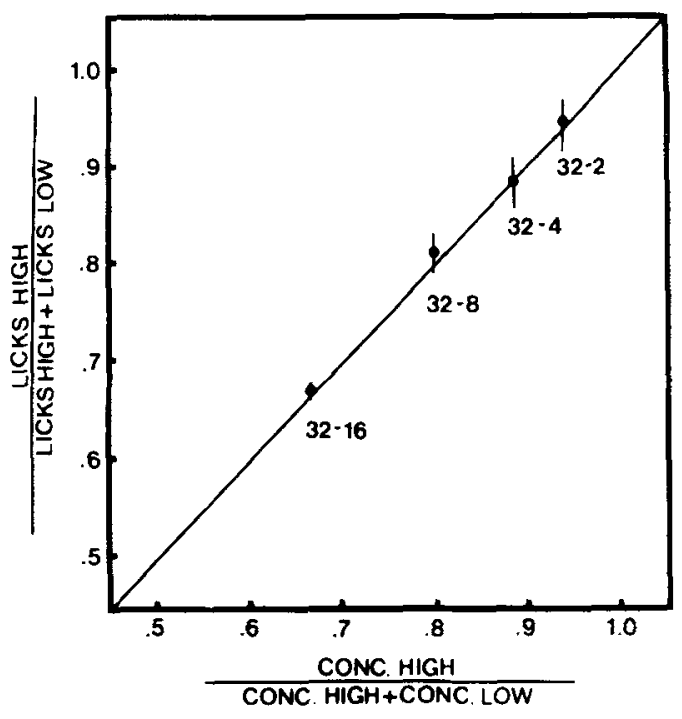

Figure 2. Proportion of licks made to the $32 \%$ tube as a function of the proportion of concentration available from that tube. Variability measures indicate the standard error of the mean.

of power functions obtained when $\log _{10}$ ratios (rather than proportions) are examined. Baum further suggested that some systematic deviations from matching might be more readily apparent in the ratio than in the proportional treatment of the data. Presented in Figure 3 is an analysis of the present data in terms of $\log _{10}$ ratios. The figure illustrates ratios obtained by dividing number of licks made for $32 \%$ by the number of licks made for the lower of each pair of concentrations. The log ratios of these lick data are then plotted as a function of the ratios of the two concentrations presented. Two sets of data are shown, one obtained on contrast days (when the two tubes contained different solutions) and one obtained on control days (when both tubes contained the same solution). The origin of the data presented in Figure 3 can perhaps be made clearer by reference to Figure 1. The data plotted as contrast data in Figure 3 represent the mean of the ratios obtained from the lick data represented in the two exterior bars from each concentration condition in Figure 1. The data plotted as control data in Figure 3 represent similar ratios derived from the two interior bars (the control conditions) of each concentration condition illustrated in Figure 1.

The best-fitting linear equations presented in Figure 3 were derived from regression analysis. It is clear that the contrast data represent a reasonable approximation to a power function $\left(\mathrm{Y}=0.92 \mathrm{X}^{1.13}\right.$ in antilog format). The data obtained from control days are not fitted quite as well by a linear function; however, the differences between the contrast and noncontrast data (the equation for the latter in nonlog form is $\mathrm{Y}=1.26 \mathrm{X}^{0.77}$ ) are interesting in regard to interpretations of contrast to be discussed below.

In addition to regression analysis, the $\log _{10}$ ratio data were subjected to a Latin square analysis of variance in order to determine whether any of the design factors influenced the animals' intake behavior. Such an analysis on the contrast data indicated an effect of concentration differences $[F(3,24)=59.08, p<.001]$ and no reliable effect of columns [test cycles, $F(3,24)=2.31, p>.05$ ] or of sequence in which the rats experienced the different sucrose pairs $[F(3,8)=1.05, p>.10]$. A similar analysis of the data obtained from control days also indicated an effect of concentration differences $[F(3,24)=24.90, p<.01]$ and no reliable effects of test cycle [columns, $F(3,24)=1.37$, $p>.10]$ or of sequences $[F(3,24)=1.33, p>.10]$. There was one complication in this analysis, in that the square-uniqueness term was statistically reliable $[F(6,24)=3.92, p<.01]$. However, using this term rather than the residual as the error term, led to no alteration in the statistical conclusions. That is, concentration effects were still reliable $[F(3,6)=$ $6.35, \mathrm{p}<.05$ ) and neither columns nor sequences were reliable [F's $<1.00$ in both cases].

One final question remains to be addressed in the presentation of the results. That is, how did degree of contrast itself vary as a function of the different

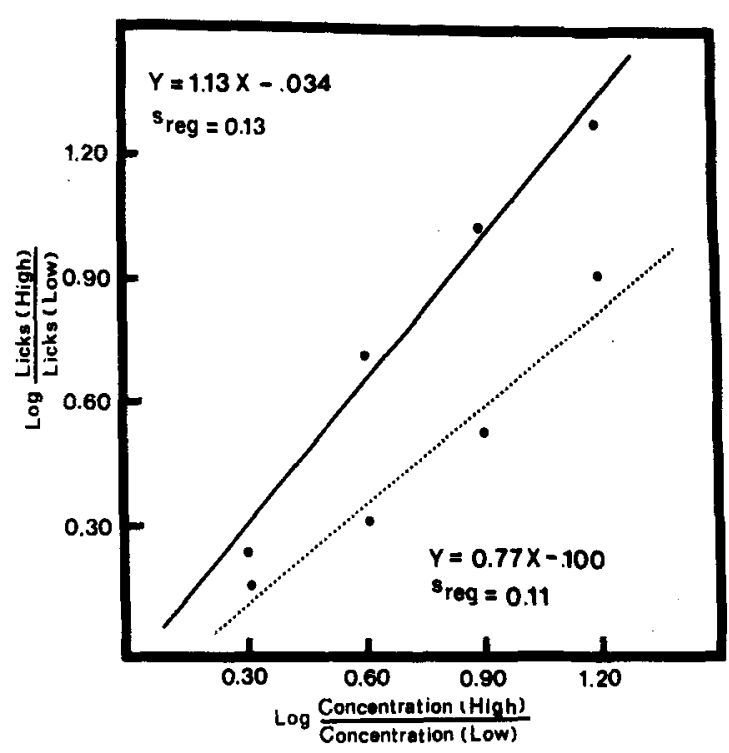

Figure 3. Regression functions obtained from ration of lick rates (licks for $32 /$ licks for 2 ; etc.) plotted as a function of the ratio of concentrations available $(32 / 2$, etc.). The upper function was obtained from contrast days, when the two tubes contained different solutions, and the lower function was obtained from control days, when the animals experienced the different concentrations between days but not within a day. The abscissa value of 0.30 represents $\log _{10}$ of $32 \% / 16 \%$, etc. 
concentration conditions? In order to take into account both positive and negative contrast and the fact that the baseline condition for negative contrast varied in the different concentration pairs, this question was addressed by the function shown in Figure 4 . In this figure, the $\log _{10}$ lick ratios obtained on contrast days are plotted as a function of the $\log _{10}$ lick ratio obtained on control days. That is, the contrast data shown in Figure 3 are plotted as a function of the control data shown in the same figure. The diagonal in this figure represents equality of the two ratios. Points above the diagonal represent an exaggeration of lick ratios under conditions in which the animals experienced two different sucrose solutions on the same day (contrast data) as compared to when the same solutions were experienced, but on different days (control data). The greater the distance above the diagonal, the greater the exaggeration (contrast). With this measure, it is apparent that contrast was greater under the 32-4 condition, slightly less with 32 vs. 2 and 32 vs. 8 , and considerably smaller with 32 vs. 16 . The nonmonotonicity of the contrast function is probably partly related to differences in the absolute rewarding properties of the different lower valued concentrations used. For example, when the lower solution is $2 \%$ sucrose, the low absolute lick rates elicited by this solution may impose limits to the degree of exaggeration possible under contrast conditions.

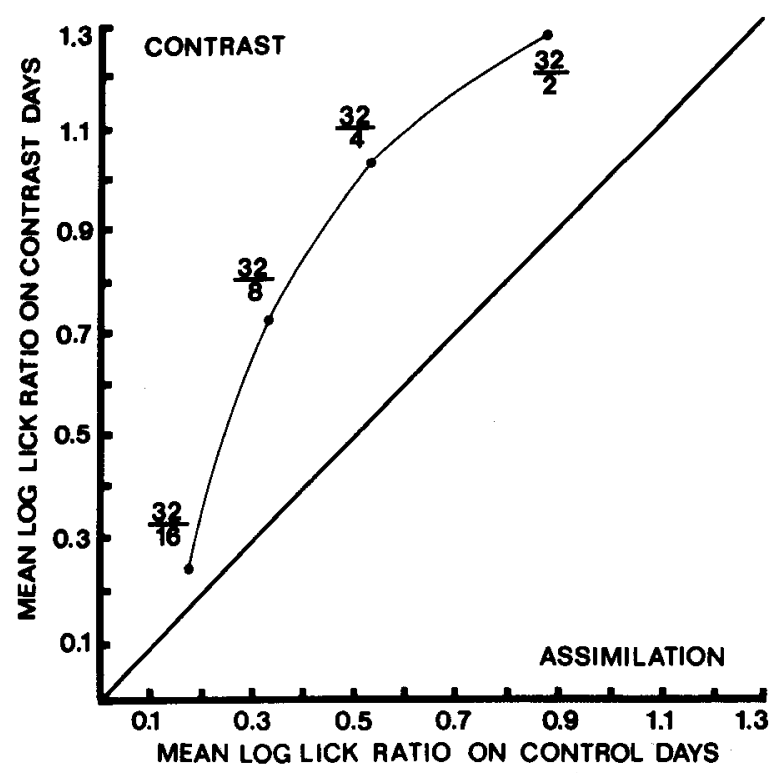

Figure 4. Ratio of licks made on contrast days plotted as a function of ratio of licks made on control days. The greater the vertical distance above the diagonal, the greater the exaggeration in lick rates produced by within-day comparisons of the two solutions. The term "assimilation" refers to the possible diminution of differences in the response to two concentrations under comparison as opposed to noncomparison conditions.
In addition to the lick data, information was gathered on the latency to initiate drinking at each switch of the tubes. These data were considerably more variable than the lick data and will not be presented in any detail. In general, a negative contrast was evident in that the animals took longer to initiate drinking when switching from a higher to a lower concentration than they did when switching between two tubes containing the lower concentration. This negative contrast tended to vary with concentration disparity in that the latency to begin drinking was longest when switching from $32 \%$ to $2 \%$, less long when switching to $4 \%$ or $8 \%$, and approximately equivalent to noncontrast conditions (16\% in both tubes) when switching from $32 \%$ to $16 \%$. There was no evidence of positive contrast in the latency data. A possible reason for the greater variability in the latency data is that, in order for this measure to reflect concentration differences, the animals must learn which concentrations are in the tubes. Since the concentration pairs changed each 4 days, there was little time for this learning to take place (see Flaherty \& Largen, 1975, for a fuller treatment of latency data under less complicated circumstances).

\section{DISCUSSION}

Contrast effects are a ubiquitous feature of both perceptual research and research on reinforcement variables. Contrast in brightness, loudness, or other perceptual domains is explained in terms of interactions within the sensing apparatus (e.g., Ratliff, 1971), or in terms of hypothetical perceptual processes (e.g., Helson, 1964), or, sometimes, when humans are used as subjects, in terms of linguistic judgment factors or memory factors (e.g., Melamed \& Thurlow, 1971; Ward \& Lockhead, 1970). Contrast obtained when amount of food reward is shifted (e.g., shift in number of food pellets) is sometimes also interpreted in terms of sensoryperceptual factors (cf. Dunham, 1968), but very often motivational processes are invoked to explain contrast obtained in these circumstances. The most frequent suggestion has been that a decrease in reward elicits an emotional response (frustration or anger) and components of this emotional response interfere with approach behavior, producing the measured contrast effect (Amsel, 1967; Bower, 1961; Cleland, Williams, \& DiLollo, 1969; Crespi, 1942, 1944; Ludvigson \& Gay, 1967). Positive contrast that occurs consequent to an increase in reward has also been interpreted in terms of the occurence of an emotional response, "elation" (Crespi, 1942).

Shifts in the concentration of sugar solutions presented to animals represent an interesting inter- 
mediate ground between shifts in amount of reward offered for the performance of a learning task and shifts in stimulus dimensions traditionally used to investigate perceptual contrast. Shifts in sugar solutions such as sucrose have, perhaps, a clearer sensory referent (Pfaffmann, 1960) than shifts in number of food pellets, and yet sugar solutions are stimuli with potent rewarding properties. The results of the present experiment are consistent with a sensory-perceptual interpretation of the contrast in consummatory behavior obtained when rats are given repeated access to different concentrations of sucrose solutions (cf. Bitterman, 1976). Our reasons for coming to this conclusion are as follows. One of the more frequently used methods in the investigation of the psychophysics of human sensory function is that of magnitude estimation. In this procedure, subjects are presented with a series of stimuli and asked to judge each proportionally to the others, or to a standard stimulus. For example, in a typical experiment investigating the relative sweetness of sugar solutions, subjects might be instructed to assign numbers in proportion to the relative sweetness of each solution (Moskowitz, 1970). The results of many experiments conducted in this fashion, on a wide variety of sensory continua, suggest that sensory intensity $(\mathrm{S})$ is related to physical intensity (I) by a power function, $\mathrm{S}=\mathrm{kI}^{n}$ (Stevens, 1969). The value of the exponent, $n$, has been found to vary with different sensory continua, with a value between 1.0 and 1.3 typical of sweetness judgments of sucrose, although lower values have sometimes been obtained (Meiselman, 1971; Moskowitz, 1971, 1973; Stevens, 1969). It is assumed that this function relates subjective sensory ratios of the judged stimuli to the physical ratios of these stimuli.

When, in the present experiment, we examined the ratio of the rats' lick rates to different concentration pairs as a function of the ratios of the concentrations themselves, we also obtained a reasonably good fit to a power function, one with an exponent of 1.13 , within the range typically found in human judgments of relative sweetness. This similarity makes it at least plausible that the rats' licking behavior in the present paradigm is being governed simply by the relative sweetness of the two sucrose solutions under immediate comparison, that is, the obtained contrast is based on sensory-perceptual mechanisms. Such an interpretation of the contrast obtained in the present paradigm would also be consistent with recent studies which have found that such contrast is uninfluenced by chlordiazepoxide, by deprivation conditions, or by imipramine, that it is not subject to disinhibition, and that such contrast will apparently endure as long as the animal is experiencing sucrose solutions of different concentrations (Flaherty, Lombardi, Kapust, \& D'Amato, 1977; Lombardi \& Flaherty, 1978; Flaherty, Wrightson, Deptula, \& Duston, Note 1).

The present data are also relevant to the extension of Herrnstein's matching law to nonoperant situations. Matching, as typically determined in operant tasks, refers to the relationship between proportion of responses made and proportion of reinforcements received in one component of a concurrent or multiple schedule. The very close matching, in terms of proportionality, obtained in the present experiment (Figure 2) occurred in a situation different in at least two important ways from the operant task. In addition to the obvious difference in the nature of the response (consummatory instead of operant), it was possible to specify the independent variable in the matching relationship (proportion of reinforcement) a priori in the present situation (proportion of concentration available), whereas in the operant paradigm this "independent" variable is typically determined a posteriori (proportion of reinforcements received). The occurrence of matching under the conditions of the present experiment seems to strengthen the claim that the phenomenon is a fundamental empirical relationship (de Villiers, 1977; Herrnstein, 1970; Killeen, 1972) rather than a definitional statement (Rachlin, 1971).

Both matching and contrast are dependent upon some degree of interaction occurring between the different reinforcers available (Herrnstein, 1970). Some indication of how contrast and matching are influenced by the time between presentation of disparate sucrose solutions may be obtained from Figure 3. The slope of the contrast function, obtained from within-session comparisons of the sucrose solutions, is steeper than the slope of the control function, which is based on between-session comparisons of the same sucrose concentrations. The contrast function tends toward "overmatching," whereas the control function tends toward "undermatching." This influence of time between comparisons on matching appears to be similar to the effects of component duration on matching obtained in operant multiple schedules; the slope of the matching function becomes steeper as the rate of alternation of the components is increased (de Villiers, 1977).

There are also data from other situations showing that contrast diminishes with time between access to different sucrose solutions. For example, Bitterman (1976) found that a contrast effect that occurred in honey bees when they were shifted from a $40 \%$ sucrose solution to a $20 \%$ sucrose solution was eliminated if as little as $24 \mathrm{~min}$ was allowed to elapse between experiences with the two solutions. 
Bitterman interpreted these data in sensory terms, attributing the loss of contrast to a recovery of taste sensitivity. Similar loss of contrast with the passage of time between successive sucrose experiences has also been shown in rats. For example, it has been found that, following 10 days exposure to $32 \%$ sucrose, the contrast that normally occurs when the animals are shifted to $4 \%$ will occur if 1,4 , or 5 days elapse since the last $32 \%$ experience, are diminished if 10 days elapse, are problematical after 17 days, and certainly gone after 32 days (Ciszewski \& Flaherty, 1977; Flaherty, Capobianco, \& Hamilton, 1973; Flaherty \& Lombardi, 1977; Gordon, Flaherty, \& Riley, 1973). It seems likely that the passage of time may influence contrast through at least two separate mechanisms. Bitterman (1976) suggested that the time factor in his honey bee experiments operated entirely through recovery of sensory processes (from adaptation to the $40 \%$ solution) and did not require the concept of memory for explanation. Similarly, it seems likely that the effects of time between comparisons evident in Figure 3 may reflect a diminished opportunity for direct sensory interaction between the two sucrose solutions under between-day test conditions as compared to withinday test conditions. However, the effects of passage of time in the successive contrast experiments cited above seem to require some statements about memory of reward since the interactions occur over such a long period of time and with the intervention of food and water consumption in the home cage. Thus, at least in the case of sucrose, it appears that there are at least two mechanisms whereby the passage of time may influence degree of interaction between two rewards (Herrnstein's ' $m$ '" parameter, 1970 ), and thereby influence degree of contrast and matching.

Finally, the method of examining degree of contrast presented in Figure 4 illustrates the logical possibility of obtaining the converse of contrast, assimilation, in some variation of the present paradigm. Assimilation, meaning a reduction in the degree of difference between two stimuli obtained under comparison, as opposed to noncomparison conditions, has been demonstrated in a number of perceptual paradigms (e.g., Parducci \& Marshall, 1962; Ward \& Lockhead, 1970; Wilson, 1972), where its relation to contrast has also been investigated. A similar line of investigation has not been pursued in the case of contrast obtained with reinforcement variables, although there is at least one study in which instrumental measures of behavior have indicated smaller differences obtained under withinsubjects variations in sucrose rewards than under between-subject variation (Ison \& Glass, 1969).

\section{REFERENCE NOTE}

1. Flaherty, C. F., Wrightson, J., Deptula, D., \& Duston, C. Conditions under which gustatory contrast is influenced by chlordiazepoxide. Manuscript submitted for publication.

\section{REFERENCES}

AMSEL, A. Partial reinforcement effects on vigor and persistence: Advances in frustration theory derived from a variety of within-subjects experiments. In K. W. Spence \& J. T. Spence (Eds.), The psychology of learning and motivation: Advances in research and theory (Vol. 2). New York: Academic Press, 1967.

Baum, W. M. On two types of deviation from the matching law: Bias and undermatching. Journal of the Experimental A nalysis of Behavior, 1974, 22, 231-242.

BitTerman, M. E. Incentive contrast in honey bees. Science, 1976, 192, 380-382.

Bower, G. H. A contrast effect in differential conditioning. Journal of Experimental Psychology, 1961, 62, 196-199.

Ciszewski, W. A., \& FlaherTy, C. F. Failure of a reinstatement treatment to influence negative contrast. American Journal of Psychology, 1977, 90, 219-229.

Cleland, E. A., Williams, M. Y., \& Dilollo, V. Magnitude of negative contrast effect in relation to drive level. Psychonomic Science, 1969, 15, 121-122.

Coren, S., \& Brussell, E. M. The effect of texture on the magnitude of simultaneous brightness contrast. Perception \& Psychophysics, 1973, 14, 277-279.

Crespr, L. P. Quantitative variation in incentive and performance in the white rat. American Journal of Psychology, 1942, 55, 467-517.

Crespi, L. P. Amount of reinforcement and level of performance. Psychological Review, 1944, 51, 341-357.

DE VILLIERs, P. Choice in concurrent schedules and a quantitative formulation of the law of effect. In W. K. Honig \& J. E. R. Staddon (Eds.), Handbook of operant behavior. Englewood Cliffs, N.J: Prentice-Hall, 1977.

DiLollo, V. Contrast effects in the judgment of lifted weights. Journal of Experimental Psychology, 1964, 68, 383-387.

Dunham, P. J. Contrasted conditions of reinforcement: A selective critique. Psychological Bulletin, 1968, 69, 295-315.

Flaherty, C. F., \& AvdzeJ, A. Bidirectional contrast as a function of rate of alternation of two sucrose solutions. Bulletin of the Psychonomic Society, 1974, 4, 505-507.

Flaherty, C. F., Capobianco, S., \& Hamilton, L. W. Effect of septal lesions on retention of negative contrast. Physiology \& Behavior, 1973, 11, 625-631.

Flaherty, C. F., \& Largen, J. Within-subjects positive and negative contrast effects in rats. Journal of Comparative and Physiological Psychology, 1975, 88, 653-664.

Flaherty, C. F., \& Lombardi, B. R. Effect of prior differential taste experience on retention of taste quality. Bulletin of the Psychonomic Society, 1977, 9, 391-394.

Flaherty, C. F., Lombardi, B. R., Kapust, J., \& D'Amato, $M$. R. Incentive contrast undiminished by extended testing, imipramine, or chlordiazepoxide. Pharmacology, Biochemistry and Behavior, 1977, 7, 315-322.

Gordon, W. C., Flaherty, C. F., \& Riley, E. P. Negative contrast as a function of the interval between preshift and postshift training. Bulletin of the Psychonomic Society, 1973, 1, 25-27.

Helson, H. Adaptation-level theory: An experimental and systematic approach to behavior. New York: Harper \& Row, 1964.

Herrnstein, R. J. On the law of effect. Journal of the Experimental Analysis of Behavior, 1970, 13, 243-266. 
Ison, J. R., \& Glass, D. H. Effects of concurrent exposure to different food and sucrose rewards in differential condi. tioning. Psychonomic Science, 1969, 15, 149-150.

KIlleEN, P. The matching law. Joumal of the Experimental Analysis of Behavior, 1972, 17, 489-495.

LOMbardi, B. R., \& Flaherty, C. F. Apparent disinhibition of successive but not of simultaneous contrast. Animal Learning \& Behavior, 1978, 6, 30-42.

Ludvigson, H. W., \& GAY, R. A. An investigation of conditions determining contrast effects in differential reward conditioning. Journal of Experimental Psychology, 1967, 75, 37-42.

Meiselman, H. L. Effect of presentation procedure on taste intensity functions. Perception \& Psychophysics, 1971, 10, 15-18.

Melamed, L. E., \& Thurlow, W. R. Analysis of contrast effects in loudness judgment. Journal of Experimental Psychology, 1971, 90, 268-274.

Mellgren, R. L. Positive and negative contrast effects using delayed reinforcement. Learning and Motivation, 1972, 3, 185-193.

Moskowitz, H. R. Ratio scales of sugar sweetness. Perception \& Psychophysics, 1970, 7, 315-320.

Moskowitz, H. R. The sweetness and pleasantness of sugars. American Journal of Psychology, 1971, 84, 387-405.
Mowkowrtz, H. R. Models of sweetness additivity. Journal of Experimental Psychology, 1973, 99, 88-98.

Parducci, A., \& Marshall, L. M. Assimilation versus contrast in the anchoring of perceptual judgments of weight. Journal of Experimental Psychology, 1962, 63, 426-437.

Pfaffman, C. The pleasures of sensation. Psychological Review, 1960, 67, 253-268.

Rachlin, H. C. On the tautology of the matching law. Journal of the Experimental Analysis of Behavior, 1971, 15, 249.251.

Ratliff, F. Contour and contrast. Proceedings of the American Philosophical Society, 1971, 115, 150-163.

Stevens, S. S. Sensory scales of taste intensity. Perception \& Psychophysics, 1969, 6, 302-307.

WARD, L. M., \& Lockhead, G. R. Sequential effects and memory in category judgments. Journal of Experimental Psychology, 1970, 84, 27.34.

WIL SON, M. Assimilation and contrast effects in visual discrimination by rhesus monkeys. Journal of Experimental Psychology, $1972,93,279.282$.

(Received for publication October 24, 1977; revision accepted March 10, 1978.) 